\title{
Losartan Protects the Heart Against Ischemia Reperfusion Injury: Sirtuin3 Involvement
}

\author{
Mohsen Sharifi Klishadi ${ }^{1}$, Farideh Zarei ${ }^{2}$, Seyyed Hassan Hejazian ${ }^{1}$, Ali Moradi ${ }^{3}$, Mahdieh Hemati ${ }^{3}$, Fatemeh Safari ${ }^{1}$ \\ ${ }^{1}$ Department of Physiology, Faculty of Medicine; ${ }^{2}$ International Campus; ${ }^{3}$ Department of Biochemistry and Molecular \\ Biology, Faculty of Medicine, Shahid Sadoughi University of Medical Sciences, Yazd, Iran.
}

Received, September 26, 2014; Revised, November 06, 2014; Accepted, February 18, 2015; Published, April 4, 2015.

\begin{abstract}
PURPOSE: Sirtuin-3 (SIRT3) deacetylase protects the heart against oxidative stress via survival factors upregulation. Clinical and experimental studies have demonstrated that activation of systemic and local renin-angiotensin system (RAS) is implicated in ischemia-induced cardiac injury. However, the relation between RAS and SIRT3 in pathophysiology of myocardial ischemia reperfusion is unknown. In this study, the cardiac transcription and expression of SIRT3 levels was examined in response to ischemia reperfusion in untreated and losartan treated rats. METHODS: Rats were divided into control group, losartan group $(\mathrm{L})$, and ischemia reperfusion (IR) groups with (L+IR) or without losatran pretreatment. Some rats were included as sham-operated and saline groups. IR was induced by left anterior descending artery occlusion. SIRT3 protein levels were determined by Western blot technique. The genes expression was specified by realtime RT-PCR. Arrhythmias were assessed according to the Lambeth conventions. RESULTS: In L+IR group a significant reduction was noted in the number of ventricular ectopic beats (VEBs) and episodes of ventricular tachycardia (VT) (VEBs: $P<0.001$; VT: $P<0.01$ vs. IR). In IR group, SIRT3 protein level was decreased in the ischemic tissue by $26.7 \pm 5.9 \%(P<0.01$ vs. Control). However, in the non-ischemic tissue the changes of SIRT3 protein content were not significant. In L+IR group SIRT3 protein levels in the ischemic part of Left ventricle were significantly different from IR group $(P<0.001)$. SIRT3 mRNA level did not change significantly among the experimental groups. Thioredoxin-1 and catalase transcription level was increased in L+IR group compared to IR group $(P<0.01)$. CONCLUSION: A decreased SIRT3 protein levels subsequent to IR might be a novel signaling mechanism involved in IR injury. Losartan at non-hypotensive dose exerts anti-ischemic effects in part by normalizing the SIRT3 protein level and upregulating the survival factors encoding genes transcription in ischemic tissue of the heart.
\end{abstract}

This article is open to POST-PUBLICATION REVIEW. Registered readers (see "For Readers") may comment by clicking on ABSTRACT on the issue's contents page.

\section{INTRODUCTION}

Reduction in the coronary blood flow during myocardial ischemia causes irreversible damage to cardiomyocytes. Restoring the blood flow or "reperfusion" is the best way to compensate for this trend. But reperfusion exacerbates the pathological mechanisms that occur during ischemia (1-2). A growing body of evidence supports the crucial role of renin-angiotensinaldosterone system (RAS) in the pathophysiology of myocardial ischemia reperfusion damage (34). Therefore, administration of angiotensin II type-1 (AT1) receptor blockers to reduce myocardial ischemia reperfusion damage is considered. Losartan is an AT1 receptor blocker that was introduced as hypotensive medication, but studies showed that apart from hemodynamic effects, it might have cardioprotective influences by affecting cardiomyocytes directly. According to previous studies, including ours, even at non- hypotensive dose, losartan may increase the ventricular fibrilation threshold and decrease infarct size, the number of premature ventricular beats, ventricular tachycardia episodes, and mortality rate (5-6-7). Losartan reduces the production of reactive oxygen species (ROS) by inhibiting NADPH oxidase enzyme (8-9) and increases the production of nitric oxide in endothelial cells by activating nitric oxide synthase (10).

Despite increasing number of studies, molecular and cellular mechanisms of myocardial ischemia reperfusion damage in the level of gene and protein are still unknown.

Corresponding Author: Fatemeh Safari Department of Physiology, Faculty of Medicine, Shahid Sadoughi University of medical sciences, Yazd, Iran. Email: Sa.physiol@gmail.com 
Situins (SIRT1-SIRT7) are a class of proteins that have attracted the attention of cardiovascular researchers in recent years. By deacetylation of histones and a wide range of non-histone proteins such as enzymes and transcription factors, sirtuins play a crucial role in the regulation of cellular biological activities such as metabolism, cell death, cell growth, and cellular responses to oxidative stress (11-12). SIRT3, which is well expressed in cardiac cells, has received a lot of attention since it is directly related to lifetime. It is a mitochondrial sirtuin that can be found in full form in the nucleus (13-14-15). Mitochondria are completely involved in producing energy and metabolism in heart and mitochondrial dysfunction is an important factor in the deterioration of ventricular contraction functions (16). SIRT3 regulates mitochondrial function by deacetylation and thereby modulation of mitochondrial proteins involved in metabolism, oxidative stress responses (17-18) and mitochondrial dynamics (19). Increased production of ROS is implicated in the development of the oxidative damage in diverse cardiovascular disease as ischemia reperfusion, hypertrophy and heart failure. SIRT3 protects the heart against oxidative stress through activation of fork head winged helix family of transcription factors, known as the fork head box O3a (FoxO3a) which increase transcription of antioxidant-encoding genes as manganese superoxide dismutase (MnSOD) and catalase (20-21). It has been shown that the hearts of SIRT3 $\%$ mice are highly susceptible to hypertrophy and fibrosis while overexpression of SIRT3 protects the cardiomyocytes against stress mediated cell death (22). Despite promising initial findings about the importance of SIRT3 and RAS in physiology and pathophysiology of the heart, there are few reports on the relationship between RAS and SIRT3 in cardiovascular system. Benigni et al. demonstrated that angiotensin-II downregulates SIRT3 mRNA and candesartan, as an AT1-blocker, prevents this effect. The longevity in AT1A-deficient mice was associated with SIRT3 upregulation. Furthermore, AT1 receptor disruption reduced cardiovascular oxidative damage in mice, which was followed by increased expression of SIRT3 in kidneys (23). Whereas there has been considerable attention directed toward the cardioprotective effects of SIRT3, the changes of SIRT3 at gene and protein levels in response to ischemia reperfusion and the possible role of RAS have not been defined. Therefore, in this study the cardiac transcription and expression of
SIRT3 protein in response to ischemia reperfusion in untreated and losartan treated rats were examined. In addition, transcription levels of catalase, Mn-SOD and thioredoxin-1, as SIRT3 target gens, were investigated.

\section{METHODS}

\section{Experimental Design:}

In this study, male Wistar rats weighing 250 to $300 \mathrm{~g}$ were used being maintained in 12-h circadian of light/dark cycle, with free access to food and water, in a temperature-controlled room. The animals were divided into the following groups: (i): Control: Intact animals without any intervention; (ii) ischemia reperfusion (IR): The left anterior descending (LAD) branch of coronary artery was occluded for $30 \mathrm{~min}$ and then reopened to perfuse the myocardium for 120 minutes; (iii) Sham-operated group: Underwent ii but without LAD occlusion; (iv) Saline: Administered saline for 4 weeks by gavage and subjected to LAD occlusion; (v) Losartan (L): Received losartan (Daru Pakhsh, Iran) 10 $\mathrm{mg} / \mathrm{kg} /$ day by gavage for 4 weeks without ischemia. Our previous study showed that pretreatment with losartan at the mentioned dose did not have hypotensive effects but reduced infract size and incidence of cardiac arrhythmia; (iv) Losratn + ischemia reperfusion $(\mathrm{L}+\mathrm{IR})$ : Pretreated with losartan underwent surgical procedure with occlusion of LAD.

\section{Rat Model of Ischemia Reperfusion}

To induce myocardial ischemia-reperfusion, animals were anesthetized by injecting $50 \mathrm{mg} / \mathrm{kg}$ of sodium thiopental and connected to Harvard ventilator at a tidal volume of $1 \mathrm{ml} / 100 \mathrm{~g}$ and a frequency of 80 inflations/min after tracheostomy. Body temperature was monitored with a rectal probe and maintained at $37^{\circ} \mathrm{C}$ using a heating pad. Arterial pressure was recorded directly via left carotid artery cannula connected to a pressure transducer. Electrocardiogram was also recorded during surgery using subcutaneous cardiography electrodes. After opening the fourth left intercostal space and removing the pericardium, 5.0 silk suture was passed below the LAD and occluded for $30 \mathrm{~min}$ by lifting the thread through a piece of polyethylene tube. Stelevation and pale downstream of LAD coronary artery demonstrated a successful ischemia that was associated with a sudden drop in blood pressure. After $30 \mathrm{~min}$ of ischemia, loosening of the suture allowed reperfusion of ischemic myocardium for $120 \mathrm{~min}$. Since the investigation 
of SIRT3 in non-ischemic tissue of the right ventricle was another goal of this study, Evans blue staining was not used. However, at the end of reperfusion, the LAD re-ligated and the ischemic tissue of left ventricle, visible by the lighter color, was immediately removed. Right ventricular tissue was also separated as nonischemic part. Tissues were washed in cold PBS solution, transferred into liquid nitrogen, and placed at $-80^{\circ} \mathrm{C}$.

\section{Reperfusion-Induced Arrhythmias}

At the beginning of the reperfusion, all animals exhibited cardiac arrhythmia. The arrhythmias were presented as the number of ventricular ectopic beats (VEBs) and episodes of ventricular tachycardia (VT). Arrhythmias were evaluated according to the diagnostic criteria in the Lambeth conventions: VEBs were discrete and identifiable premature QRS complex, VT was defined as a run of four or more consecutive VEBs. VEBs included bigeminy and salvos as well (24).

\section{Western Blot Analysis}

Changes in SIRT3 protein level were measured by Western blot technique. For this purpose, protein concentration was determined after homogenization (lysis buffer solution contained: $150 \mathrm{mM}$ Nacl, $1 \%$ Triton $\mathrm{X}-10,0.25 \%$ sodium deoxycholate, $0.1 \%$ SDS, $50 \mathrm{mM}$ Tris-HCL, $1 \mathrm{mM}$ EDTA, 2mM EGTA, $20 \mathrm{mM}$ HEPES and 2mM PMSF) using the Bradford method at a wavelength of $590 \mathrm{~nm}$. Before loading, the samples were incubated in sample buffer contained glycerol, SDS, bromophenol blue, 2mercaptoethanol, and Tris- $\mathrm{HCl}$ for $7 \mathrm{~min}$ at $100^{\circ}$ C. After preparing SDS-PAGE gel at a concentration of $10 \%$, equal amount of each sample was loaded and electrophoresed. In order to compare protein expression among the experimental groups, the samples of different groups were run on the same gel. Protein bands in the gel were transferred to polyvinylidene fluoride membrane (Amersham Bioscience Co., UK) and then blocking was performed by $2 \%$ skimmed milk powder and $1 \%$ bovine serum albumin in Tris-buffered saline with Tween (TBST) (1mM Tris-HCl, pH 7.6, $1.5 \mathrm{mM} \mathrm{NaCl}$, $0.1 \%$ Tween-20). SIRT3 was incubated by polyclonal rabbit anti-SIRT3 primary antibody (Santa Cruz Biotechnology Inc, USA) and then secondary antibody of goat anti-rabbit IgG-HRP (Santa Cruz Biotechnology Inc, USA). The primary antibody detects short length of SIRT3 protein $(28 \mathrm{kD})$. $\beta$-actin protein was used as a reference for comparing changes in SIRT3 protein (primary antibody: Santa Cruz Biotechnology Inc, USA). In the final step, chemiluminescent was added to blotting and the locations of SIRT3 or $\beta$-actin protein bands were determined using a gel documentation instrument (Syngene GBOX, 680X).

\section{Quantitative Reverse Transcription Polymerase Chain reaction}

Based on the manufacturer's instructions, total RNA was extracted ( RNeasy fibrous tissue mini kit from QIAGEN, USA ). By measuring the absorbance at $260 \mathrm{~nm}$, concentrations of RNA were identified and its purity was evaluated by 260/280 nm absorbance ratio (Eppendorf, Hamburg, Germany). Using random hexamers, dNTP and Moloneymurine leukemia virus reverse transcriptase (Fermentas), in a total volume of $20 \mu \mathrm{L}, 1 \mu \mathrm{g}$ of total RNAs was applied to synthesize first strand cDNA. Quantitative RTPCR analysis was performed in triplicates using SYBR-green in the Rotor Gene system (Corbett Research 2004, Australia). Normalization was achieved against $\beta$-actin and relative quantity of gene expression was analyzed based on the Pfaffl method. Following are the nucleotide sequences of the PCR primers: SIRT3: forward 5'TGCACGG TCTGTCGAAGGTC-3 and reverse 5'-ATGTC AGGTTTCACAACGCCAGTA-3' (25), Thiore-doxi-1: forward 5'TTCCTTGAAGTAG ACGTGGATGAC-3' and reverse 5'-AGAGA ACTCCCCAACCTTTTGAC-3', Catalase: forward 5'-CTGACGTCCACCCTGACT $-3^{\prime}$ and reverse 5'-GGCAGCTATGTGAGAGCC-3', $\beta$ actin: forward 5'-GAACCCTAAGGCC AACCGTGAAAAGAT-3' and reverse 5'ACCGCTCGTTGCCAATAGTGATG-3'.

\section{STATISTICAL ANALYSIS}

To compare the hemodynamic parameters at baseline vs. $4 \mathrm{~min}$ after LAD occlusion within the same rat, paired t-test was used. Comparisons between the groups were done by ANOVA. A $P$ value $<0.05$ was considered as statistically significant. Statistical analysis was performed using Prism software ( $n=7$ in each group).

\section{Ethics Approval}

All experimental procedures were performed in accordance with the approved instructions of the Ethics Committee on Animal Experiments of Shahid Sadoughi University, Yazd, Iran. 


\section{RESULTS}

\section{Effects of Losartan on Hemodynamic Parameters}

As shown in Table 1, mean arterial pressure, directly recorded from the carotid before LAD occlusion, was not significantly different among the experimental groups. Immediately after LAD occlusion, arterial blood pressure decreased significant in all groups. However, at the end of reperfusion, blood pressure was nearly close to the baseline. Therefore, there was no significant difference in the blood pressure at sampling time among the experimental groups.

\section{Effects of Losartan on Reperfusion-induced Arrhythmias}

In our previous study, the effects of losartan at a dose of $10 \mathrm{mg} / \mathrm{kg} / \mathrm{day}$ on the infarct size and ischemia-induced arrhythmias have been reported. The aim of this study was to investigate changes in SIRT3 at gene and protein levels, so infarct size was not measured. However, given that electrocardiogram was recorded in all samples, data relating to arrhythmias during reperfusion were also considered and analyzed. In ischemia reperfusion with $30 \mathrm{~min}$ of ischemia and $120 \mathrm{~min}$ of reperfusion, with the beginning of reperfusion, many arrhythmias appeared in ECG that were examined. Figure 1 shows that the number of ventricular ectopic beats in L+IR group have significantly decreased compared to IR group (IR: $359.1 \pm 22$; L+IR: 211.8 $\pm 20, p<$ $0.001)$. In addition, reduced number of episodes of ventricular tachycardia in L+IR group was meaningful compared to IR group (IR: 34.22 \pm 3.4 ; L+IR: $23.6 \pm 2.6, p<0.01$ ).
Effects of Ischemia Reperfusion on SIRT3 Transcription and Expression in Right and Left Ventricles. One of the main purposes of this study was to investigate the effects of myocardial ischemia reperfusion on transcription and expression of SIRT3 protein. Figure 2 shows that in response to regional ischemia reperfusion, in the ischemic area of left ventricle, the SIRT3 protein level reached $26.7 \pm 5.9 \%$ that indicated a significant reduction compared to control group $(p<0.01)$.

To understand if possible changes in gene and protein expression level were limited to ischemic area or the whole heart, SIRT3 at mRNA and protein levels were measured in nonischemic tissue of right ventricle as well. Figure 2 represents that in non-ischemic tissue of right ventricle in IR group, SIRT3 protein level was decreased but this reduction was not statistically significant compared to control group.

In another part of this study, changes in the rate of SIRT3 gene transcription were examined. As shown in Figure 3, SIRT3 mRNA level did not show significant changes in IR group compared to control group nor in the ischemic area of left ventricle neither in the non-ischemic tissue of right ventricle.

Effects of Losartan on SIRT3 Protein and mRNA Levels in Right and Left Ventricles in Response to Ischemia Reperfusion

To further explore the possible mechanisms for the anti-ischemic effect of losartan, SIRT3 protein and mRNA levels were assessed in losartan treated animals subjected to IR. As shown in Figure 2, in the ischemic ventricle of L+IR group SIRT3 protein levels reached $84.6 \pm 21.9 \%$ which was not significantly different from control group.

Table 1. Hemodynamic parameters.

\begin{tabular}{lcccccc}
\hline & \multicolumn{2}{c}{ Baseline } & \multicolumn{2}{c}{4 min post-LAD occlusion } & \multicolumn{2}{c}{ End of reperfusion } \\
\hline Group & MAP & HR & MAP & HR & MAP & HR \\
Sham & $118 \pm 9$ & $382 \pm 16$ & $101.4 \pm 6.1$ & $409 \pm 21$ & $110 \pm 8.5$ & $391 \pm 18$ \\
Saline & $103 \pm 10$ & $411 \pm 21$ & $71 \pm 5.9^{\mathrm{b}}$ & $435 \pm 19$ & $112 \pm 6^{\mathrm{d}}$ & $397 \pm 16$ \\
IR & $113 \pm 4$ & $387 \pm 19$ & $76.1 \pm 4.3^{\mathrm{a}}$ & $421 \pm 12$ & $109 \pm 5.7^{\mathrm{c}}$ & $403 \pm 13$ \\
L+IR & $107 \pm 8$ & $398 \pm 17$ & $78.2 \pm 5.2^{\mathrm{a}}$ & $414 \pm 8$ & $112 \pm 4.1^{\mathrm{d}}$ & $375 \pm 12$ \\
L & $110 \pm 6.4$ & $401 \pm 11$ & NM & NM & NM & NM \\
\hline
\end{tabular}

Mean arterial pressure (MAP) ( $\mathrm{mmHg}$ ) and heart rate (HR) (beats/min) obtained at baseline, 4 min after left anterior descending (LAD) artery occlusion and at the sampling time in rats subjected to ischemia reperfusion (IR) with or without losartan (L). In sham group, MAP was measured 4 min after the tread was passed below the LAD. In L group the LAD occlusion was not induced therefore the MAP was not measured (NM). In all of rats subjected to IR, blood pressure decreased significantly after LAD occlusion but following reperfusion, it was nearly close to the baseline. Data are expressed as Mean \pm S.E.M. ${ }^{\mathrm{a}} p<0.05,{ }^{\mathrm{b}} p<0.01$ compared with baseline values within the same rat. ${ }^{\mathrm{c}} p<0.05,{ }^{\mathrm{d}} p<0.01$ compared with 4 -min post LAD occlusion time within the same rat. 
However, meaningful difference was observed compared to IR group $(p<0.001)$ Two-way ANOVA analysis indicates that the interaction between two interventions of treatment and ischemia reperfusion was statistically significant in SIRT3 protein $(p<0.001)$. In the right ventricle of L+IR group no important changes were observed in SIRT3 protein levels.
In rats receiving only losartan and did not underwent ischemia reperfusion (L group), SIRT3 protein level did not show a significant difference compared to the control group neither in left nor in right ventricle.

In groups $\mathrm{L}$ and $\mathrm{L}+\mathrm{IR}$ no significant changes were observed in SIRT3 mRNA levels neither in the ischemic area of the left ventricle nor in the non-ischemic tissue of right ventricle (Figure 3).
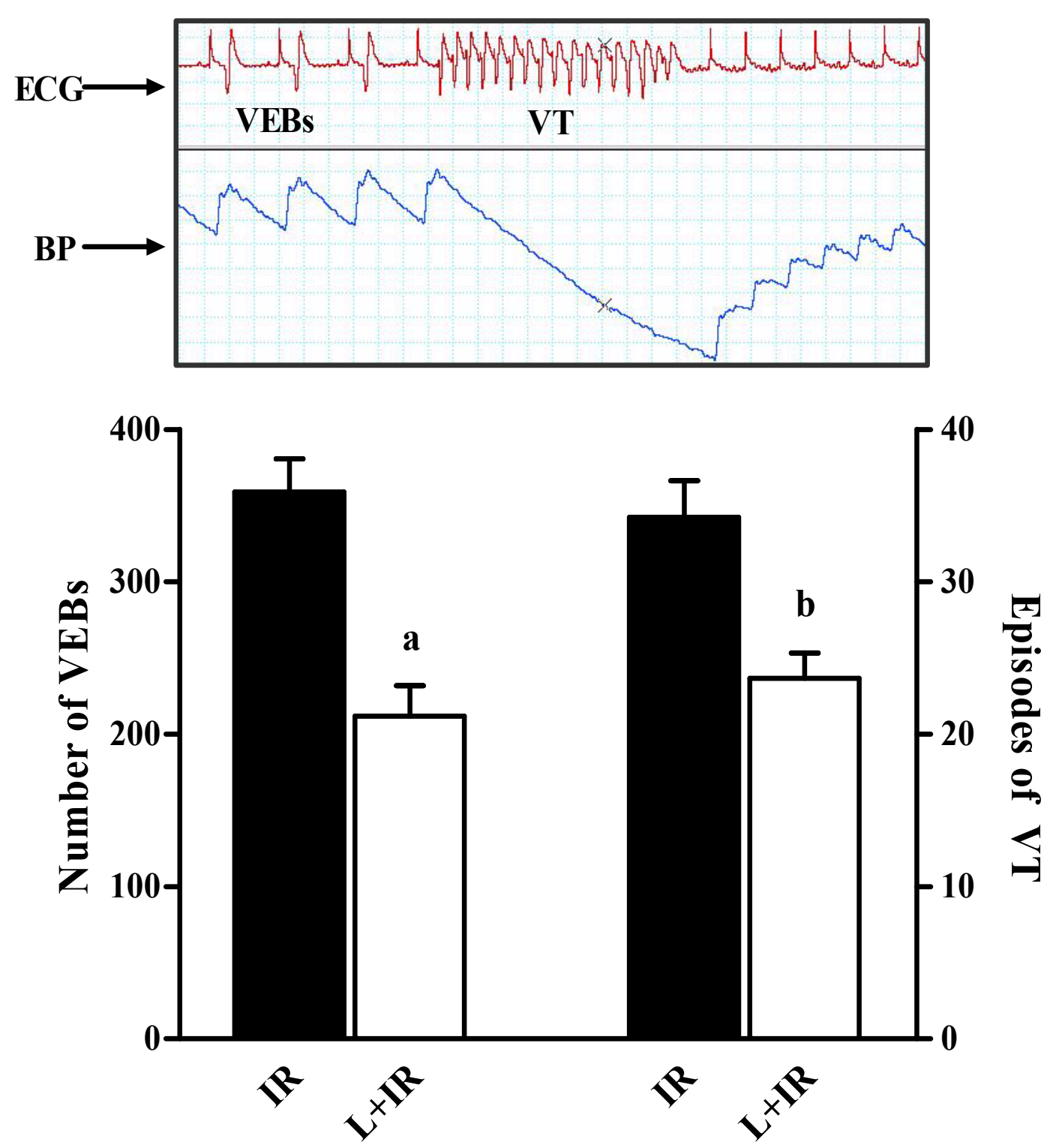

Figure 1. Reperfusion-induced arrhythmias. The number of ventricular ectopic beats (VEBs) and episodes of ventricular tachycardia (VT) during reperfusion in rats subjected to ischemia reperfusion (IR) with or without losartan (L). A sample of recorded ECG at the beginning of reperfusion is shown at top of the panel. All data are expressed as Mean \pm S.E.M. ${ }^{\mathrm{a}} p<0.001,{ }^{\mathrm{b}} p<0.01$ compared to IR group. 


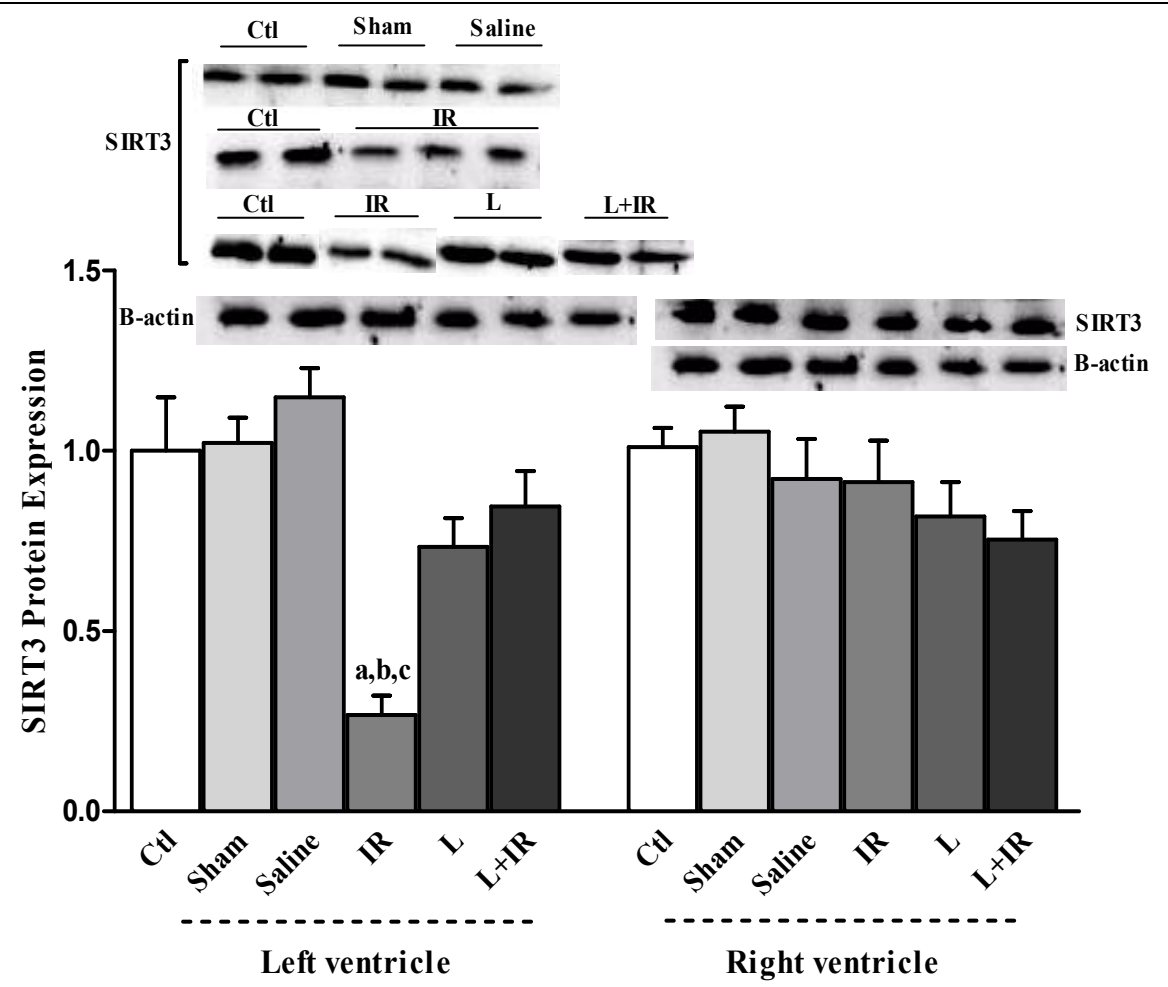

Figure 2. Cardiac SIRT3 protein expression. Expression of SIRT3 protein in the ischemic left ventricle and non-ischemic right ventricle of control animals $(\mathrm{Ctl})$ and rats subjected to ischemia reperfusion (IR) with or without losartan (L) pretreatment. $\beta$-actin was used as loading control. All data are expressed as Mean \pm S.E.M. ${ }^{\mathrm{a}} p<0.01 v s . \mathrm{Ctl},{ }^{\mathrm{b}} p<0.05 v s$. $\mathrm{L}$ and ${ }^{\mathrm{c}} p<0.001 v s$. L+IR group.

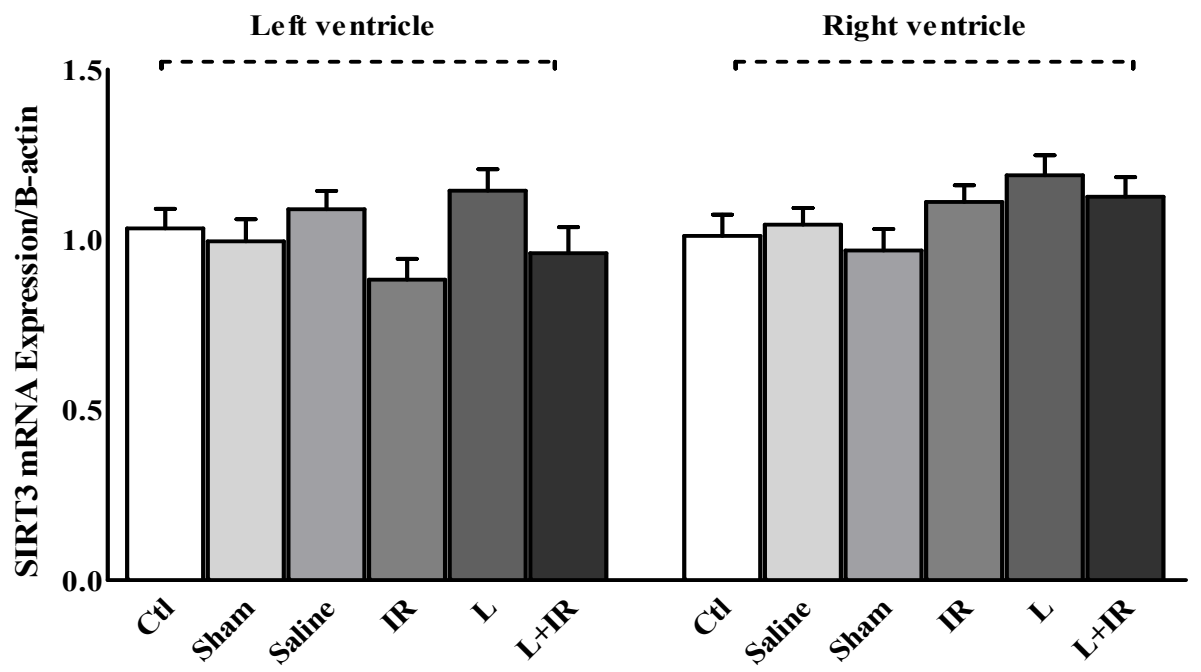

Figure 3. Cardiac transcription levels of SIRT3. SIRT3 mRNA levels in ischemic area of left ventricle and non-ischemic tissue of right ventricle in rats treated with losartan (L) with or without ischemia reperfusion (IR). All data are expressed as Mean \pm S.E.M.

\section{Survival Genes Transcription Levels:}

As shown in Figure 4, in response to ischemia reperfusion, the transcription level of catalase, Mn-SOD and thioredoxin1-encoding genes increased but not statistically significant.
However, catalase and thioredoxin- 1 mRNA levels increased in L+IR group by $45.7 \pm 3.2 \%$ and $57.6 \pm 4 \%$, respectively which was significant in comparison with control $(p<0.01)$ and IR $(p<$ $0.05)$ groups. 

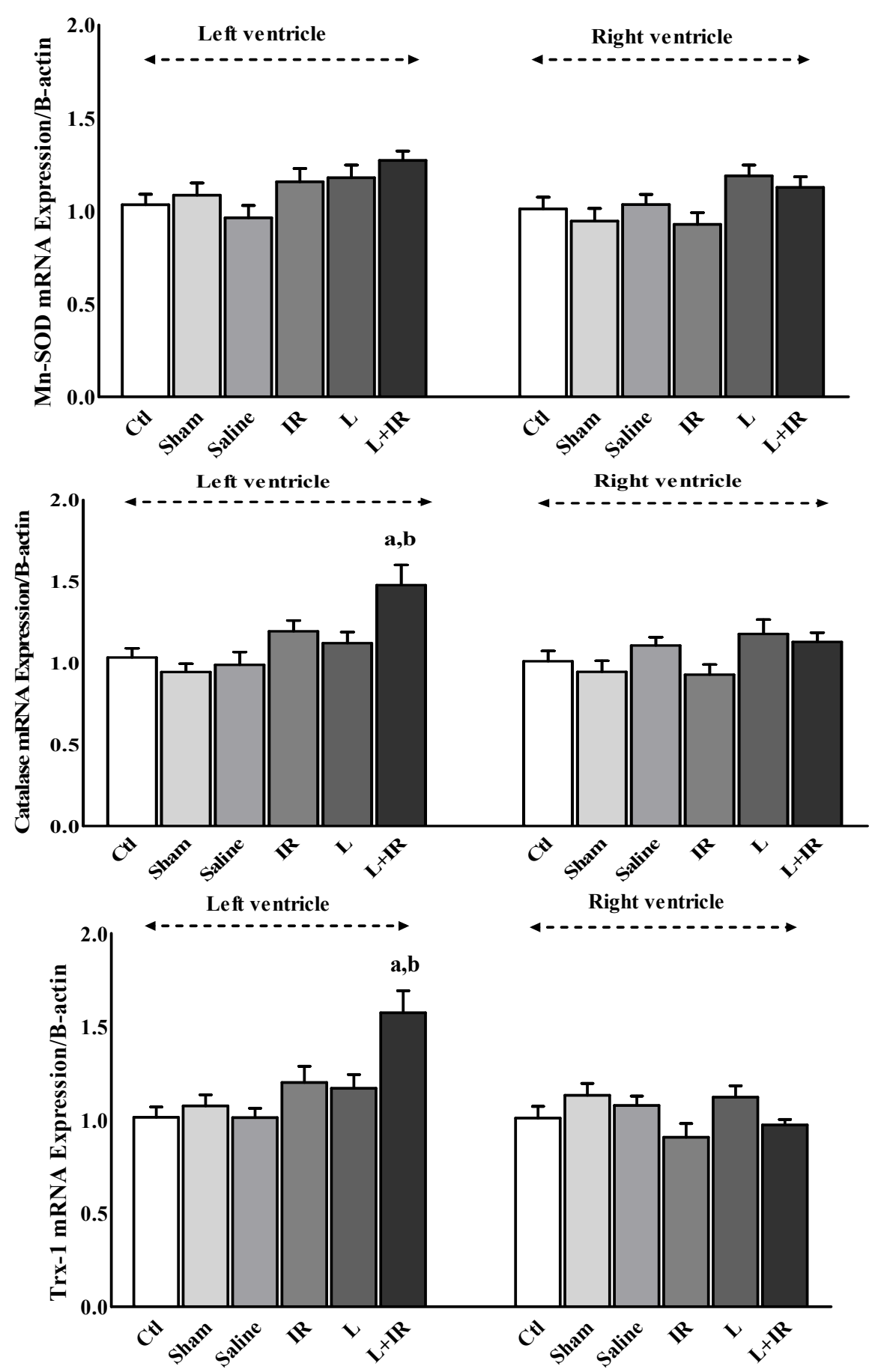

Figure 4. Cardiac transcription levels of survival factors-coding genes. Relative expression of $M n$-SOD, catalase and thioredoxin-1 genes as the cardiac survival factors in the ischemic area of left ventricle and non-ischemic tissue of right ventricle in rats treated with losartan (L) with or without ischemia reperfusion (IR). Results were normalized to $\beta$-actin expression. All data are expressed as Mean \pm S.E.M. ${ }^{a} p<0.01$ compared to Ctl. and ${ }^{b} p<0.05$ compared to IR group.

\section{DISCUSSION}

Mounting evidence implicates the crucial cardioprotective roles for SIRT3. However, the effect of acute regional ischemia reperfusion on the protein and mRNA levels of this key cardiac deacetylase and the possible role of RAS as the critical component in pathophysiology of ischemia reperfusion is unknown. The results of the present study indicated that during acute 
myocardial ischemia reperfusion, SIRT3 protein levels in left ventricular ischemic tissue reduced. Administration of anti-ischemic dose of losartan, by itself, did not increase SIRT3 protein content, however, normalized SIRT3 protein level in ischemic tissue of the heart.

SIRT3 is a mitochondrial and cytosolic deacetylase that is present in cytosol in full length at the molecular weight of $44 \mathrm{kDa}$ and in mitochondria in short length at the molecular weight of $28 \mathrm{kDa}$. When SIRT3 enters into mitochondria, 142 amino acids are detached from $\mathrm{N}$-terminal by matrix processing peptidase and it is converted to the short active form. Studies have shown that both forms of the enzyme are functional and show enzymatic activity (15-26). SIRT3 regulates mitochondrial function by deacetylation of mitochondrial proteins. In SIRT3 $^{-}$hearts mitochondrial proteins are hyperacetylated. Mitochondrial dysfunction is well proven to be associated with ischemia reperfusion injury, especially when cells are exposed to abundant ROS production during reperfusion. Opening mitochondrial permeability transition pore, during ischemia and reperfusion, causes the release of apoptotic molecules and cell death $(27,28)$. By deacetylation of cyclophilin D, as a regulatory part of this complex, SIRT3 prevents cardiac mitochondrial dysfunction (29). It also activates antioxidant enzyme superoxide dismutase (SOD2), a major mitochondrial antioxidant enzyme, by deacetylating two lysine residues in it, so that ROS are reduced and oxidative stress damage is prevented. Furthermore, by deacetylating the transcription factor of FoxO3a, SIRT3 increases its migration into the nucleus. After translocation FoxO3a by increasing the expression of survival factors encoding genes such as MnSOD, catalase, Bcl2 and peroxiredoxin, diminishes ROS production in cardiomyocytes thereby protects the heart against oxidative injury $(20,21)$. On the other hand, by activating FoxO3a, MAPK/ERK and PI3K/Akt pathways that are important signaling pathways of myocardial stress-induced damage are inhibited (20). Mukherjee et al. have demonstrated that longevinex (modified SIRT1 activator) improves cardiac performance and decreases infarct size in hearts subjected to 30 min of ischemia followed by $2 \mathrm{~h}$ reperfusion while increases survival factors expression and theses effects were accompanied by enhanced SIRT3 expression (30). In another study resveratrol, SIRT1 activator, protected the heart against ischemia reperfusion injury via activation of Foxo3a subsequent to SIRT3 activation which increased intracellular concentration of glutathione in the heart (31).

In this study, after acute myocardial ischemia reperfusion the levels of SIRT3 protein decreased in left ventricular ischemic tissue suggesting that SIRT3 downregulation might be a mechanism of cardiomyocytes damage in response to ischemia reperfusion. To evaluate the "local" or "systemic" nature of the effect of ischemia on SIRT3, the protein was measured in nonischemic tissue of the right ventricle. The results demonstrated that in right ventricle, in contrast to ischemic area of the left ventricle, no significant reduction was observed in protein levels. In other words, the effect of ischemia on SIRT3 protein level is a local effect limited to the area of ischemia. Since SIRT3 is considered as an important factor in cardio protection, the results of our study suggested that reduction in SIRT3 in the cardiac ischemic tissue at least served as one of the mechanisms of ischemia reperfusion injury. This observation was supported by studies showing that SIRT3 had potential to suppress ischemia reperfusion injury. For example, Porter et al. investigated the role of SIRT3 in mediating the response to myocardial ischemia reperfusion and showed that SIRT3 $\left({ }^{+} /\right)$hearts were more vulnerable to ischemia reperfusion and showed less functional recovery and greater infarct size suggested decreased SIRT3 increases the susceptibility of hearts to ischemia reperfusion damage (32). Furthermore, SIRT3 has been shown to protect the heart against transverse aortic constriction-induced hypertrophy during aging (29). The change of SIRT3 in response to oxidative stress was investigated by Sundaresan et al. They evaluated the levels of short and long forms of SIRT3 protein in response to different cardiac stressors and revealed that hydrogen peroxide (oxidative stress inducer), angiotensin II and phenylepherin elevated the levels of longform SIRT3 in cardiomyocytes. Even though, a significant change in the level of the short form of SIRT3 after cellular stress was not detected (22). It should be pointed that in their study the protein levels were measured 24 or $48 \mathrm{~h}$ after treatment the cardiomyocytes with genotoxic or oxidative stress inducers. Furthermore, the nature of the in vitro stress situation was different from the in vivo ischemia reperfusion-induced stress investigated in our study. Sirtuins, including SIRT1 and SIRT3, are considered as stressresponse enzymes, therefore, in response to myocardial ischemia reperfusion, SIRT3 was expected to increase in compensation. However, in our study, acute myocardial ischemia 
reperfusion led to decrease in SIRT3 protein, which may be due to different mechanisms. Since considering the ischemic time, protein level was measured 150 min after LAD occlusion, it cannot be decided if protein level increased merely because of ischemia or early reperfusion. Another finding of the present study was the disparity between mRNA and protein. Interestingly, there was no reduction in the level of SIRT3 mRNA of ischemic tissue, which may partly reflect the lack of change in the rate of transcription of SIRT3 in ischemic tissue. It should also be pointed out that in our study the mRNA as well as the protein was measured at the sampling time (150 min after LAD occlusion), therefore, early increase and then degradation of mRNA before the sampling time was supposed. The disparity between mRNA and protein is a phenomenon, which is observed in physiological and pathological conditions. For example, our group reported the discordance between uncoupling protein-2 (UCP2) mRNA and protein in the left ventricle following ischemia reperfusion previously. In another study, we measured the UCP2 and UCP3 mRNA levels in response to ischemia followed by different duration of reperfusion and showed that increase of mitochondrial UCP2 but not UCP3 protein was independent of transcription and other mechanisms were involved (33). FertBober et al. showed that in ischemic hearts with or without reperfusion proteins levels increased but the total mRNA levels diminished (34). Therefore, we think that measurement of SIRT3 at mRNA and protein levels immediately after ischemia and at the beginning of reperfusion provides valuable findings to accept or reject this hypothesis. Other mechanisms such as post transcription mechanisms or even protein degradation may be responsible for SIRT3 mRNA and protein disparity. Finding the exact mechanism requires studies that are more detailed. There are reports stating that SIRT3 migrates to mitochondria in stressful situations, deacetylates proteins such as acetyl CoA syntethase-2, and help improve mitochondrial function $(17,18)$. Since in the current study the mitochondrial content of SIRT3 was not measured and changes were related to the total protein, we cannot judge about the mitochondrial content of SIRT3.

SIRT3 is a NAD-dependent deacetylase; conditions, which increase NAD level, activate SIRT3 and consequently target deacetylation paths. That is why it is called "NAD sensor" ( 15 , 26). During myocardial reperfusion ischemia, ROS-induced damage leads to the production of the DNA single-strand breaks which increases catabolism of NAD, resulting in reduced level of NAD (35). Therefore, exploring the possibility of reduction in SIRT3, which activities in response to myocardial ischemia reperfusion can also result in valuable findings.

In our study, administration of losartan at a dose of $10 \mathrm{mg} / \mathrm{kg} /$ day could considerably reduce the severity of arrhythmias during reperfusion while there was no significant change in animal's blood pressure. In other words, the effect of losartan on reperfusion arrhythmias can be created independent from hypotensive effect of this medication. This result confirms previous findings suggesting the reduction in the infarct size and arrhythmias by losartan. An interesting point in this study was that in animals, which received losartan SIRT3 protein level was not decreased significantly in ischemic tissue. In other words, losartan prevented the reduction in the SIRT3 protein level of ischemic myocardial tissue. There is not enough information available about the relationship between RAS and sirtuins, especially in heart. Bening et al. showed the relationship between RAS and sirtuins in cultured epithelial cells. In these cells angiotensin II downregulated SIRT3 mRNA level while AT1 receptor blockade inhibited this effect. On the other hand, activating SIRT1 by resveratrol has managed to down-regulate AT1 receptor at mRNA and protein levels. Therefore, some of the effects of sirtuins activators may also be due to inhibiting RAS system (23). The results of the current study revealed that AT1 receptor blockage normalized SIRT3 protein level in the ischemic heart suggesting RAS activation could be a mechanism of SIRT3 protein level regulation in response to ischemia reperfusion in the heart. In other words, AT1 blocker as losartan increases SIRT3 protein levels as a protective mechanism toward lowering the cardiac ischemia reperfusion damage. Providing an accurate model of RAS and SIRT3 relationship requires a series of careful and extensive experiments. The transcription of Trx-1, Mn-SOD and catalase was assessed, considering the importance of prosurvival factors in cardioprotection against IR injury. Trx-1 is a redox-regulating protein of cardiomyocytes, playing a key role in augmenting cardiomyocytes resistance against oxidative stress which is diminished during IR. Preconditioning experiments protect heart against ischemia through increasing expression of Trx-1. Those mice who overexpress Trx-1 are resistant to IR injury, $(36,37)$ while inhibition of Trx-1 causes cardiac hypertrophy (38). Catalase is the most 
important enzyme involved in decomposition/detoxification of $\mathrm{H}_{2} \mathrm{O}_{2}$ thereby increases cardiac resistance to oxidative stressinduced injury such as ischemia reperfusion. Therefore, in this study, the transcription of aforementioned prosurvival factors was evaluated .

As demonstrated in Figure 4, losartan augmented transcription of Trx-1 and Mn-SOD in ischemic ventricle. SIRT3 increases the transcription of survival factors via activation of transcription factor, FOXO3. In our study, the reduction of SIRT3 was not associated with decreased transcription of survival genes. However, augmentation of mentioned genes transcription in ischemic ventricle in response to losartan was associated with the increase in SIRT3 protein level. Therefore, losartan may magnify SIRT3 protein actions in ischemic tissue; although the direct action of losartan on cardiomyocytes should be considered.

Based on the findings of the current study, it can be suggested that during ischemia reperfusion, when myocardium is exposed to an influx of ROS, some unknown factors reduce SIRT3 protein levels in the left ventricle; as a result, the heart will be more prone to injury. However, losartan can prevent the reduction of SIRT3 protein in ischemic heart by either inhibiting these paths or direct effects, consequently makes the heart more resistant against damage.

\section{ACKNOWLEDGEMENT}

This article was extracted from a Master's thesis in Medical Physiology by the author, Mohsen Sharifi Klishadi and was supported by Shahid Sadoughi University of Medical Sciences, Yazd, Iran. The Authors would like to thank Dr. Naghdi for providing losartan and Ms. Zahra Anvari for her expert technical assistance.

\section{REFERENCES}

1. Hausenloy DJ. and Yellon DM. Myocardial ischemia-reperfusion injury: a neglected therapeutic target. J Clin Invest, 123:92-100, 2013.

2. Dorweiler B., Pruefer D., Andrasi TB., Maksan SM., Schmiedt W., Neufang A., Vah CF. Ischemia-reperfusion injury Patophysiology and clinical implications. Eur J Trauma Emerg Surg,33:600-612, 2007.

3. Yang BC., Philips MI., Ambuehl PE., Shen BS. Increase in angiotensin II type 1 receptor expression immediately after ischemia- reperfusion in isolated rat hearts. Circulation, 96:922-926, 1997.

4. Baker KM., Booz GW., Dostal DE. Cardiac actions of angiotensin II: role of an intracardiacrenin-angiotensin system. Annu Rev Physiol, 54:227-241, 1992.

5. Sladek T., Sladkova J., Kolar F., Papousek F., Cicutti N., Korecky B., Rakusan K. The effect of AT1 receptor antagonist on chronic cardiac response to coronary artery ligation in rats. Cardiovasc Res, 31:568-76, 1996.

6. Safari F., Hajizadeh S., Shekarforoush S., Bayat G., Foadoddini M., Khoshbaten A. Influence of ramiprilat and losartan on ischemia reperfusion injury in rat hearts. J Renin-AngiotensinAldosterone Syst, 13:29-35, 2012.

7. Zhu B., Sun Y., Sievers RE., Browne AE., Pulukurthy S., Sudhir K., Lee RJ., Chou TM., Chatterjee K., Parmley WW. Comparative effects of pretreatment with captopril and losartan on cardiovascular protection in a rat model of ischemia-reperfusion. J Am Coll Cardiol, 35:787-95, 2000.

8. Mollnau H., Wendt M., Szöcs K., Lassègue B., Schulz E., Oelze M., Li H., Bodenschatz M., August M., Kleschyov AL., Tsilimingas N., Walter U., Förstermann U., Meinertz T., Griendling K., Münzel T.Effects of angiotensin II infusion on the expression and function of NAD (P) $\mathrm{H}$ oxidase and components of nitric oxide/cGMP signaling. Circ Res,90:E58-65, 2002.

9. Safari F., Hajiadeh S., MoshtaghionSM., Forouzandeh Moghadam M., Shekarforoush S., BayatG., Mazlum R., SattarianL.Effect of losartan on NOX2 transcription following acute myocardial ischemia-reperfusion. Physiol Pharmacol , 16:44-53, 2012.

10. Messadi-Laribi E., Griol-Charhbili V., Pizard A., Vincent MP., Heudes D., Meneton P., AlhencGelas F., Richer C. Tissue kallikrein is involved in the cardioprotective effect of AT1-receptor blockade in acute myocardial ischemia. J Pharmacol Exp Ther, 323:210-216, 2007.

11. Kelly GS. A review of the sirtuin system, its clinical implications, and the potential role of dietary activators like resveratrol: part 2, Altern Med Rev, 15:313-328, 2010.

12. Lavu S., Boss O., Elliott PJ., Lambert PD. Sirtuins-novel therapeutic targets to treat ageassociated diseases. Nat Rev Drug Discov, 7:841853, 2008.

13. Pillai VB., Sundaresan NR., Jeevanandam V., Gupta MP. Mitochondrial SIRT3 and heart disease. Cardiovasc Res, 88:250-256, 2010.

14. Jin L., Galonek H., Israelian K., Choy W., Morrison M., Xia Y.,Wang X., Xu Y., Yang Y., Smith JJ., Hoffmann E., Carney DP., Perni RB., Jirousek MR., Bemis JE., Milne JC., Sinclair DA., Westphal CH. Biochemical characterization, localization, and tissue distribution of the longer 
form of mouse SIRT3. Protein Sci,18:514-525, 2009.

15. Onyango P., Celic I., McCaffery JM., Boeke JD., Feinberg AP. SIRT3, a human SIR2 homologue, is an NAD-dependent deacetylase localized to mitochondria. Proc Natl Acad Sci U S A, 15:13653-8, 2002.

16. Tompkins AJ., Burwell LS., Digerness SB., Zaragoza C., Holman WL., Brookes PS. Mitochondrial dysfunction in cardiac ischemiareperfusion injury. ROS from complex I, without inhibition. Biochim Biophys Acta, 1762:223-231, 2006.

17. Newman JC., He W., Verdin E. Mitochondrial protein acylation and intermediary metabolism: regulation by sirtuins and implications for metabolic disease. J Biol Chem, 287:42436-43, 2012.

18. Bharathi SS., Zhang Y., Mohsen AW., Uppala R., Balasubramani M., Schreiber E., Uechi G., Beck ME., Rardin MJ., Vockley J., Verdin E., Gibson BW., Hirschey MD., Goetzman ES. Sirtuin 3 (SIRT3) protein regulates long-chain acyl-CoA dehydrogenase by deacetylating conserved lysines near the active site. J Biol Chem, 288:33837-47, 2013.

19. Samant SA., Zhang HJ., Hong Z., Pillai VB., Sundaresan NR., Wolfgeher D., Archer SL., Chan DC., Gupta MP. SIRT3 deacetylates and activates OPA1 to regulate mitochondrial dynamics during stress.Mol Cell Biol, 34:807-19, 2014.

20. Sundaresan NR., Gupta M., Kim G., Rajamohan SB., Isbatan A., Gupta MP. Sirt3 blocks the cardiac hypertrophic response by augmenting Foxo3a-dependent antioxidant defense mechanisms in mice. J Clin Invest, 119:2758, 2009.

21. Qiu X., Brown K., Hirschey MD., Verdin E., Chen D. Calorie restriction reduces oxidative stress by SIRT3-mediated SOD2 activation. Cell Metab, 12:662-7, 2010.

22. Sundaresan NR., Samant SA., Pillai VB., Rajamohan SB., Gupta MP. SIRT3 is a stressresponsive deacetylase in cardiomyocytes that protects cells from stress-mediated cell death by deacetylation of $\mathrm{Ku} 70$. Mol Cell Biol, 28:6384401, 2008.

23. Benigni A., Corna D., Zoja C., Sonzogni A., Latini R., Salio M., Conti S., Rottoli D., Longaretti L., Cassis P., Morigi M., Coffman TM., Remuzzi G. Disruption of the Ang II type 1 receptor promotes longevity in mice. J Clin Invest, 119:524-530, 2009.

24. Curtis MJ., Hancox JC., Farkas A., Wainwright CL., Stables CL., Saint DA., Clements-Jewery H., Lambiase PD., Billman GE., Janse MJ., Pugsley MK., Ng GA., Roden DM., Camm AJ., Walker MJ.The Lambeth Conventions (II): guidelines for the study of animal and human ventricular and supraventricular arrhythmias. Pharmacol Ther, 139:213-248, 2013.
25. Zeng L., Yang Y., Hu Y., Sun Y., Du Z., Xie Z., Zhou T., Kong W. Age-related decrease in the mitochondrial sirtuin deacetylase Sirt3 expression associated with ROS accumulation in the auditory cortex of the mimetic aging rat model. PLoS One, 9:e88019, 2014.

26. Schwer B., North BJ., Frye RA., Ott M., Verdin E. The human silent information regulator (Sir)2 homologue hSIRT3 is a mitochondrial nicotinamide adenine dinucleotide-dependent deacetylase. J Cell Biol, 158:647-657, 2002.

27. Orrenius S., Gogvadze V., Zhivotovsky B. Mitochondrial oxidative stress implications for cell death. Annu Rev Pharmacol Toxicol, 47: 14383, 2007.

28. Tompkins AJ., Burwell LS., Digerness SB., Zaragoza C., Holman WL., Brookes PS.Mitochondrial dysfunction in cardiac ischemia-reperfusion injury. ROS from complex I, without inhibition. Biochim Biophys Acta, 1762: 223-31, 2006.

29. Hafner AV., Dai J., Gomes AP., Xiao CY., Palmeira CM., Rosenzweig A., Sinclair DA. Regulation of the mPTP by SIRT3-mediated deacetylation of CypD at lysine 166 suppresses age-related cardiac hypertrophy. Aging (Albany NY), 2:914-23, 2010.

30. Mukherjee S., Ray D., Lekli I., Bak I., Tosaki A., Das DK. Effects of Longevinex (modified resveratrol) on cardioprotection and its mechanisms of action. Can J Physiol Pharmacol. 88:1017-25, 2010.

31. Das S., Mitrovsky G., Vasanthi HR., Das DK. Anti aging properties of a grape-derived antioxidant are regulated by mitochondrial balance of fusion and fission leading to mitophagy triggered by a signaling network of Sirt1-Sirt3-Foxo3-PINK1-PARKIN. Oxid Med Cell Longev,2014:345105, 2014

32. Porter GA., Urciuoli WR., Brookes PS., Nadtochiy SM. SIRT3 deficiency exacerbates ischemia-reperfusion injury: implication for aged hearts. Am J Physiol Heart Circ Physiol, 306:H1602-9, 2014.

33. Safari F., Anvari Z., Moshtaghioun S., Javan M., Bayat G., Foroshs S., Hekmatimoghaddam S. Differential expression of cardiac uncoupling proteins 2 and 3 in response to myocardial ischemia-reperfusion in rats.Life Sci, 98:68-74, 2014.

34. Fert-Bober J., Basran RS., Sawicka J., Sawicki G. Effect of duration of ischemia on myocardial proteome in ischemia/reperfusion injury. Proteomics, 8:2543-55, 2008.

35. Lindahl T., Satoh MS., Poirier GG., Klungland A. Posttranslational modification of poly (ADPribose) polymerase induced by DNA single strand breaks. Trends Biochem Sci, 20: 405-411, 1995.

36. Turoczi T, Chang VW, Engelman RM, Maulik N, Ho YS, Das DK. Thioredoxin redox signaling in the ischemic heart: an insight with transgenic 
mice overexpressing Trx1. J Mol Cell Cardiol 35:695-704, 2003.

37. Das DK.Thioredoxin regulation of ischemic preconditioning. Antioxid Redox Signal. 6:40512,2004
38. Yamamoto M,Yang G, Hong C, Liu J, Holle E, $\mathrm{Yu} \mathrm{X}$ et al.Inhibition of endogenous thioredoxin in the heart increases oxidative stress and cardiac hypertrophy. J Clin Invest 112:1395-406, 2003. 